\title{
A FURTHER THEOREM OF THE SYLVESTER TYPE
}

\author{
M. EDELSTEIN, F. HERZOG AND L. M. KELLY
}

1. Introduction. In [2] the following theorem is established:

THEOREM I. If $\left\{S_{i}\right\}$ is a finite collection of disjoint, bounded, closed sets in $E_{n}$ at least one of which is infinite, and not all of which are subsets of the same line, then there exists a line cutting exactly two of the sets.

On the other hand Edelstein [1] proved:

THEOREM II. Let $\left\{S_{i}\right\}$ be a finite collection of sets in $E_{n}$, nonempty, mutually exclusive, closed and bounded and not all subsets of the same line. Let every set of the collection have a finite number of components and let at least one of the sets of $\left\{S_{i}\right\}$ have a component which is not a single point. Then there exists a hyperplane cutting exactly two of the sets.

Theorem II has both substantially stronger hypotheses and a stronger conclusion. The present paper obtains the stronger conclusion of Theorem II from the weaker hypotheses of Theorem I. For a more elaborate bibliography and historical comment on problems of the Sylvester type see [2], [3], and [4].

Precisely what the situation is when the requirement that one of the sets be infinite is dropped is not known. (See [2, Theorem 4.1].) This matter is still under investigation.

2. Admissible directions. We consider the euclidean $n$-space $E_{n}$. Any $(n-1)$-dimensional linear subspace of $E_{n}$ will be referred to as a hyperplane, the surface $x_{1}^{2}+x_{2}^{2}+\cdots+x_{n}^{2}=1$ as the unit hypersphere.

If $\Sigma$ is any compact point set in $E_{n}$ there corresponds to each direction $\gamma$ (realized as a unit vector or as a point on the unit hypersphere) a unique support hyperplane $\pi$ of $\Sigma$, which is (a) perpendicular to $\gamma$, and (b) such that the perpendicular direction from $\pi$ to any point of $\Sigma$ which is not on $\pi$ is the same as (rather than opposite to) the direction of $\gamma$. The correspondence between all directions $\gamma$ and all support hyperplanes $\pi$ of $\Sigma$ is in general bi-unique. Only when all of $\Sigma$ lies in one hyperplane of $E_{n}$ there exists at least one pair of opposite directions to which the same support hyperplane $\pi$ corresponds.

Let $\Sigma \equiv \bigcup_{i=1}^{p} S_{i}$, where each $S_{i}$ is compact and $S_{i} \cap S_{j}=\varnothing$ for $i \neq j$. We shall call a direction $\gamma$ an admissible direction (with respect to the partition $\Sigma \equiv S_{1} \cup S_{2} \cup \cdots \cup S_{p}$ ) if the corresponding support hyper-

Received by the editors February 13, 1962. 
plane $\pi$ of $\Sigma$ intersects only one of the sets $S_{i}$. If $\pi$ intersects more than one of these sets we call $\gamma$ an inadmissible direction.

Lemma 1. The set of all admissible directions with respect to the partition $\Sigma \equiv \bigcup_{i=1}^{p} S_{i}$ is open and everywhere dense on the unit hypersphere.

Proof. Let $\gamma_{0}$ be an admissible direction. Let $\pi_{0}$ be the support hyperplane of $\Sigma$ which corresponds to $\gamma_{0}$ and let $\pi_{0}$ intersect $S_{k}$. Then all points of $\Sigma-S_{k}$ lie on one side of $\pi_{0}$ but not on $\pi_{0}$. Let $P_{k}$ be an arbitrary point of $\pi_{0} \cap S_{k}$. The line connecting any point of $\Sigma-S_{k}$ with $P_{k}$ forms an angle $\alpha$ with $\pi_{0}$, where $0<\alpha \leqq 90^{\circ}$. If $\alpha_{0}$ is the minimum of all these values $\alpha$ then $\alpha_{0}>0$, and it is clear that any direction $\gamma$ making an angle less than $\alpha_{0}$ with $\gamma_{0}$ will also be an admissible direction. The set of admissible directions is therefore open.

It remains to show that in any neighborhood of a given direction $\gamma_{1}$ there is an admissible direction. It suffices to assume that $\gamma_{1}$ is an inadmissible direction. Let $\pi_{1}$ be the support hyperplane of $\Sigma$ corresponding to $\gamma_{1}$; the set $\pi_{1} \cap \Sigma$ then contains points from more than one of the sets $S_{i}$. Let $q$ be an $(n-2)$-dimensional linear variety in $\pi_{1}$ which is a support for $\pi_{1} \cap \Sigma$ and intersects $\pi_{1} \cap \Sigma$ in a single point, say $P_{j} \in S_{j}$. (Such a support $q$ can be obtained, for instance, by choosing an arbitrary point $O$ in $\pi_{1}, O \notin \pi_{1} \cap \Sigma$. Let $P_{j}$ be a point of $\pi_{1} \cap \Sigma$ at maximal distance from $O$, and let $q$ be perpendicular to the line $O P_{j}$ and pass through the point $P_{j}$.). If now $\pi_{1}$ is rotated about $q$ in the proper direction through a sufficiently small angle, the resulting hyperplane will intersect $S_{j}$ only and, therefore, will be parallel to a support hyperplane $\pi$ which intersects $S_{j}$ only. The direction $\gamma$ corresponding to $\pi$ is admissible and arbitrarily close to $\gamma_{1}$.

3. The main theorem. For the proof of the main theorem, we need the following Lemma 2, in which the notion of a pseudo-support is used. This notion was introduced in $[2$, p. 328] and will be repeated here for the sake of completeness.

Definition. A hyperplane in $E_{n}$ which intersects a point-set $S$ and is such that at least one of the open half-spaces defined by that hyperplane contains at most a finite number of points of $S$ is a pseudosupport of $S$.

Leмma 2. If $\left\{S_{i}\right\}$ is a finite collection of two or more mutually disjoint compact sets in $E_{n}, n \geqq 2$, with $\Sigma=\bigcup S_{i}$ infinite and if none of the sets $\Sigma-S_{i}$ lies in a hyperplane, then there exist an index $k, a$ point $P_{k} \in S_{k}^{\prime}$, the derived set of $S_{k}$, an $(n-2)$-dimensional linear variety $l$ through $P_{k}$, and a hyperplane $\pi$ containing $l$ with the following properties: 
(i) $\pi$ is a pseudo-support for $\Sigma-S_{k}$;

(ii) $l$ contains no points of $\Sigma$ other than $P_{k}$;

(iii) at least one of the half-spaces of $\pi$ bounded by $l$ is free of points of $\left(\Sigma-S_{k}\right)^{\prime}$;

(iv) there exists a sequence of points $\left\{Q_{r}\right\}$ with $Q_{r} \in S_{k}, Q_{r} \notin \pi$ and $\lim Q_{r}=P_{k}$.

Proof. We begin by choosing a support hyperplane $\sigma$ of $\Sigma^{\prime}$ which intersects $\Sigma^{\prime}$ in a single point, say $P_{k} \in S_{k}$. Consider now an arbitrary $(n-2)$-dimensional linear variety $l$ in $\sigma$ through $P_{k}$, subject only to the restriction that $l$ should contain no point of $\Sigma$ other than $P_{k}$. This is evidently possible since there are at most denumerably many points of $\Sigma$ on $\sigma$. We remark that this restriction on the choice of $l$ will, in the procedure which follows, ensure that in any consideration of the approach of $P_{k}$ by points of $S_{k}$, the possibility that such an approach might be possible only along $l$ is certainly eliminated.

We now distinguish two cases:

Case (a). There are points of $\Sigma-S_{k}$ on $\sigma$. If $P_{k}$ can be approached by points of $S_{k}$ not on $\sigma$ then $\sigma$ has all desired properties, and we take $\pi=\sigma$. If $P_{k}$ can be approached only by points of $S_{k}$ on $\sigma$ then rotate $\sigma$ about $l$ in either direction. By the hypothesis of the lemma there must be points of $\Sigma-S_{k}$ not on $\sigma$. Hence if we denote by $\pi$ the first hyperplane obtained in the rotation that intersects $\Sigma-S_{k}$, then $\pi \neq \sigma$ and $\pi$ will satisfy all the requirements of the lemma.

Case (b). There are no points of $\Sigma-S_{k}$ on $\sigma$. Rotate $\sigma$ about $l$ in either direction. Let $\tau$ be the first hyperplane obtained in this way that intersects $\Sigma-S_{k}$. If $P_{k}$ can be approached by points of $S_{k}$ not on $\tau$, then take $\pi=\tau$. If, however, $P_{k}$ can only be approached by points of $S_{k}$ on $\tau$, then return to $\sigma$ and rotate it in the opposite direction about $l$. Let the first hyperplane that intersects $\Sigma-S_{k}$ be $\pi$. Since not all of $\Sigma-S_{k}$ can lie on $\tau$, we must have $\pi \neq \tau$, and $\pi$ will now have the properties desired in the lemima.

THEOREM III. Let $\left\{S_{i}\right\}$ be a finite collection of three or more mutually disjoint compact sets in $E_{n}$ with $\Sigma=U S_{i}$ infinite. Then either $\Sigma$ is a subset of a line or there exists a hyperplane intersecting exactly two of the sets $S_{i}$.

Proof. The case $n=2$ is taken care of by [2, Theorem 2.1]. Assume the theorem valid for $E_{n-1}(n \geqq 3)$ and let $\Sigma$ be a nonlinear set. We first dispose of the case in which one of the sets $\Sigma-S_{i}$, say $\Sigma-S_{h}$, lies in some hyperplane $\rho$, in which case Lenma 2 does not apply. If $S_{h}$ also lies in $\rho^{*}$ then $\Sigma \subset \rho$ and (by induction) an $(n-2)$-dimensional linear variety $\lambda$ in $\rho$ exists which intersects exactly two of the sets 
$S_{i}$. Hence any hyperplane (in $E_{n}$ ) containing $\lambda$ and not containing $\rho$ will do the same.

If $S_{h}$ has points not on $\rho$, let $q$ be an $(n-2)$-dimensional linear variety in $\rho$ which intersects exactly one of the sets of $\Sigma-S_{h}$. (For instance let $q$ be a support, relative to $\rho$ as a containing space, of $\Sigma-S_{h}$, intersecting $\Sigma-S_{h}$ in a single point.) Now any hyperplane through $q$ and a point of $S_{h}$ not on $\rho$ will intersect exactly two of the sets $S_{i}$.

We thus assume that none of the sets $\Sigma-S_{i}$ lies in a hyperplane and apply Lemma 2 . Let then $\pi$ and $l$ satisfy Lemma 2 . We think of the hyperplane $\pi$ as "horizontal" and assume that there are at most finitely many points of $\Sigma-S_{k}$ "above" $\pi$. Furthermore, the $(n-2)$-dimensional linear variety $l$ divides $\pi$ into two half-hyperplanes of which the "left" one, say, will be assumed to be free of points of $\left(\Sigma-S_{k}\right)^{\prime}$.

Under the assumption that both directions perpendicular to $l$ in $\pi$ are admissible directions (in the sense of $\$ 2$ ) with respect to each of the following partitions:

$$
\begin{gathered}
\pi \cap\left(\Sigma-S_{k}\right)=\bigcup_{i \neq k}\left(\pi \cap S_{i}\right), \\
\pi_{r} \cap\left(\Sigma-S_{k}\right)=\bigcup_{i \neq k}\left(\pi_{r} \cap S_{i}\right),
\end{gathered}
$$

where $\pi_{r}$ denotes the open right half-hyperplane of $\pi$ bounded by $l$, we denote $l$ by $l^{*}$. (If $\pi_{r} \cap\left(\Sigma-S_{k}\right)=\varnothing$ the second of the above partitions should, of course, be disregarded.) If $l$ does not satisfy these conditions we consider a new $(n-2)$-dimensional linear variety $l^{*}$ chosen as follows.

On the unit hypersphere in $\pi$ we surround the two ends $\gamma_{1}$ and $\gamma_{2}$ of the diameter, the direction of which is perpendicular to $l$, by neighborhoods $N_{1}$ and $N_{2}$ respectively.

If $N_{1}$ and $N_{2}$ are sufficiently small then, clearly, any hyperplane through $P_{k}$ and perpendicular to $\gamma_{3} \in N_{1} \cup N_{2}$ will be disjoint from $\Sigma-S_{k}$.

Lemma 1, together with the obvious fact that the intersection of finitely many sets, each of which is open and everywhere dense on the unit hypersphere is itself everywhere dense, ensures that a direction $\gamma^{*} \in N_{1} \cup N_{2}$ exists such that $\gamma^{*}$ together with the direction opposite to $\gamma^{*}$ are both admissible with respect to the above two partitions. We take $l^{*}$ perpendicular to $\gamma^{*}$ and passing through $P_{k}$.

It must be noted, however, that this variety $l^{*}$, if $l^{*} \neq l$, does not 
necessarily satisfy any more the condition (ii) of Lemma 2. But $l^{*}$ contains no points of $\Sigma-S_{k}$, which is enough for our purposes.

We now distinguish between two cases (not mutually exclusive).

Case (a). There exists a sequence of points $\left\{Q_{n}\right\}$ with $Q_{n} \in S_{k}$, $\lim Q_{n}=P_{k}$ and $Q_{n}$ above $\pi$. Let $m$ be the support, relative to $\pi$, of $\pi \cap\left(\Sigma-S_{k}\right)$ which is parallel to $l^{*}$ and such that all of $\pi \cap\left(\Sigma-S_{k}\right)$ lies on the same side of $m$ as $l^{*}$. (There are either two such supports or only one, depending on whether there are points of $\pi \cap\left(\Sigma-S_{k}\right)$ on both sides of $l^{*}$ or not.)

The support $m$ intersects exactly one of the sets, say $S_{j}, j \neq k$, and it is clear that, for sufficiently large $n$, the hyperplane through $m$ and $Q_{n}$ intersects $S_{j}$ and $S_{k}$ but no other $S_{i}$.

Case (b). There exists a sequence of points $\left\{Q_{n}\right\}$ with $Q_{n} \in S_{k}$, $\lim Q_{n}=P_{k}$ and $Q_{n}$ below $\pi$.

(i) If $\pi_{r} \cap\left(\Sigma-S_{k}\right) \neq \varnothing$ let $m$ be the support of $\pi_{r} \cap\left(\Sigma-S_{k}\right)$ which is parallel to $l^{*}$ and such that no point of $\pi_{r} \cap\left(\Sigma-S_{k}\right)$ lies between $m$ and $l^{*}$. As above, let $m$ intersect the set $S_{j}, j \neq k$. Since there are no points of $\left(\Sigma-S_{k}\right)^{\prime}$ to the left of $l^{*}$ on $\pi$, we can again conclude that, for large $n$, the hyperplane through $m$ and $Q_{n}$ intersects $S_{j}$ and $S_{k}$ but no other $S_{i}$.

(ii) If $\pi_{r} \cap\left(\Sigma-S_{k}\right)=\varnothing$ let $m$ be any $(n-2)$-dimensional linear variety in $\pi$ parallel to $l^{*}$, intersecting exactly one set $S_{j}, j \neq k$; for instance, let $m$ be a support of $\pi \cap\left(\Sigma-S_{k}\right)$ parallel to $l^{*}$. In this case there are no points of $\left(\Sigma-S_{k}\right)^{\prime}$ on $\pi$ and hence, for large $n$, the hyperplane through $m$ and $Q_{n}$ intersects $S_{j}$ and $S_{k}$ but not $S_{i}, i \neq j, i \neq k$.

\section{BIBLIOGRAPHY}

1. M. Edelstein, $A$ further generalization of a problem of Sylvester, Riveon Lematematika 11 (1957), 50-55.

2. F. Herzog and L. M. Kelly, A generalization of the theorem of Sylvester, Proc. Amer. Math. Soc. 11 (1960), 327-331.

3. L. M. Kelly and W. O. J. Moser, On the number of ordinary lines determined by $n$ points, Canad. J. Math. 10 (1958), 210-219.

4. T. Motzkin, The lines and planes connecting the points of a finite set, Trans. Amer. Math. Soc. 70 (1951), 451-464.

Michigan State University and Technion, Haifa, IsRael 